\title{
Optimum low complexity filter bank for generalized orthogonal frequency division multiplexing
}

\author{
Mohammad Hadi Abbaszadeh', Babak H. Khalaj ${ }^{2,3^{*}}$ and Afrooz Haghbin ${ }^{1}$
}

\begin{abstract}
Generalized frequency division multiplexing (GFDM) is one of the multicarrier modulation candidates proposed for the 5th generation of wireless networks. Among GFDM linear receivers, GFDM MMSE receiver achieves the best error performance for multipath fading channels at the cost of high numerical complexity. Hence, the combination of GFDM match filter (MF) receiver and double-side successive interference cancellation (DSIC) method is used instead. However, there is a significant gap between the error performance of GFDM MMSE and DSIC/MF receivers for the case of employing modern channel coding. Recently, we have proposed a new multicarrier scheme based on GFDM architecture called generalized orthogonal frequency division multiplexing (GOFDM). This study derives an optimized cyclic tree-structured perfect reconstruction-quadrature mirror filter (PR-QMF) bank for GOFDM transceiver and then introduces a novel method for implementation of the optimum filter bank in the frequency domain. Employing such a fast and optimum filter bank provides several advantages for GOFDM transceiver. GOFDM transmitter mitigates out-of-band spectrum leak to the level of that of GFDM. In addition, choosing an appropriate configuration of filter bank yields lower peak to average power ratio in transmit signal of GOFDM compared to that of OFDM. On the other hand, while GOFDM MMSE receiver has lower numerical complexity compared with GFDM DSIC/MF receiver, its coded bit error rate curve is close to that of GFDM MMSE receiver. The aforementioned advantages envision GOFDM as a competitive candidate to be employed in the physical layer of new wireless applications.
\end{abstract}

\section{Introduction}

Spectrum is a limited media for growing demand of radio communications, and hence, spectrum efficiency is one of the important parameters to be taken into account for any modulation proposed as the air interface of the 5th generation (5G) wireless networks. Low computational complexity of the transceiver algorithm is another important parameter especially for use cases that require low battery consumption. OFDM has been widely used in wireless transmission due to its low computational complexity and good performance for multipath channels $[1,2]$. In order to provide the possibility of using a simple one-tap frequency domain equalizer

\footnotetext{
* Correspondence: khalaj@sharif.edu

2Department of Electrical Engineering, Sharif University of Technology, Tehran, Iran

${ }^{3}$ School of Computer Science, Institute for Research in Fundamental Sciences (IPM), Tehran, Iran

Full list of author information is available at the end of the article
}

(FDE) at the receiver side of OFDM, cyclic prefix (CP) is generally added to OFDM symbols. Since CP length should, at least, be equal to the channel length, $\mathrm{CP}$ overhead ratio is high for large channel spreads. Moreover, OFDM suffers from high out-of-band spectrum leak [3] which forces many subcarriers to be left vacant in order to fit the OFDM spectrum in an emission spectrum mask. Therefore, OFDM achieves low spectral efficiency due to large guard time and guard band and thus could not be recommended as an appropriate choice for new wireless applications.

Among the alternative filtered multicarrier schemes [4, 5], general frequency division multiplexing (GFDM) is known as a versatile multicarrier modulation being able to address many of the requirements of new wireless applications [6]. GFDM was first introduced in [7] and then has been widely investigated in the 5 GNOW project $[8,9]$. Three common linear receivers, i.e., zero-forcing (ZF), 
matched filter (MF), and minimum mean square error (MMSE) receivers, are evaluated for the receiver side of GFDM. Among GFDM linear receivers, GFDM MMSE receiver yields the lowest error rate at the cost of the highest complexity which makes it unusable for many applications [10]. Therefore, matched filter (MF) receiver in combination with the double-side successive interference cancellation (DSIC) algorithm is used instead to provide a trade-off between computational complexity and bit error rate (BER) performance [11].

On the basis of GFDM architecture, we have recently introduced a generic cyclic filtered multicarrier scheme called extended-GFDM. Employing perfect reconstructionquadrature mirror filter (PR-QMF) bank in the generic scheme yields generalized orthogonal frequency division multiplexing (GOFDM). GOFDM benefits from architectural advantages of GFDM including flexibility of the structure, high spectral efficiency, and low latency. We have shown GOFDM MMSE outperforms GFDM ZF receiver in terms of error performance on the assumption of perfect synchronization. In addition, we have proved GOFDM MMSE receiver has a simple structure that consists of onetap MMSE-FDE prior to PR-QMF receiver filter bank [12].

The first contribution of this paper is to propose an optimized tree-structured PR-QMF bank in order to achieve low out-of-band (OOB) spectrum emission. Simulation results show that employing proposed optimized PR-QMF bank in GOFDM structure results in OOB spectrum leak as low as that of GFDM transmitter. In addition, it is shown GFDM and GOFDM transmitters which employ an appropriate configuration of filter bank can improve peak to average power ratio (PAPR) compared with that of OFDM transmit signal. As the second contribution, a novel method for implementing the cyclic tree-structured filter bank in the frequency domain is introduced. Such implementation results in a lower computational complexity for GOFDM MMSE receiver compared with existing implementation techniques proposed for GFDM DSIC/MF receiver under multipath fading channels. Further, it will be seen GOFDM MMSE receiver yields better error performance compared with OFDM for multipath fading channel where modern error control coding is presented in the setup. In the similar conditions, GFDM encounters complexity and error performance challenges for MMSE and DSIC/MF receivers, respectively. The remaining parts of the paper are organized as follows. In
Section 2, the extended-GFDM scheme is described. In Section 3, the first optimum low-complexity PR-QMF bank is derived and then the GOFDM transceiver block diagram based on such filter bank is introduced. Simulation results presented in Section 4 compare GOFDM and GFDM in terms of spectrum localization, computational complexity, error performance, and PAPR considering OFDM as a benchmark. The paper is concluded in Section 5.

\section{Extended-GFDM scheme}

Figure 1 illustrates a general form of baseband multicarrier modulation inspired from GFDM architecture; therefore, it is called extended-GFDM scheme. At the transmitter side, $\boldsymbol{x}_{t i}(n)$ for $i=1: K$ are $K$ blocks of $M$ complex symbols generated by a QAM mapper. Up-sampling $\boldsymbol{x}_{t i}(n)$ sequences by the factor of $K$ generates $\boldsymbol{x}_{t i}^{e}(n)$ sequences of $N=M K$ symbols whose symbols are given as

$$
x_{t i}^{e}(n)= \begin{cases}x_{t i}\left(\frac{n}{K}\right), n=0, K, 2 K, \ldots,(M-1) K \\ 0 \quad \text { otherwise. }\end{cases}
$$

Assume $g_{i}(n)$ and $h_{i}(n)$ for $i=1: K$ to be impulse responses of transmitter and receiver filters with length less than or equal to $N$, we have

$$
\boldsymbol{y}(n)=\sum_{i=1}^{K} \boldsymbol{x}_{t i}^{e}(n) \stackrel{N}{\circledast} g_{i}(n),
$$

where $\circledast N$ denotes $N$-point circular convolution. Substituting for $x_{t i}^{e}(n)$ symbols from (1) yields $y(n)$ symbols as

$$
y(n)=\sum_{i=1}^{K} \sum_{j=0}^{M-1} x_{t i}(j) \tilde{g}_{i}(n-j K) \text { for } n=0: N-1,
$$

where $\tilde{g}_{i}(n-j K)$ is achieved through $j K$ cyclic shift of $g_{i}(n)$ after extending to $N$ symbols through zero padding. Next, inserting $\mathrm{CP}$ at the beginning of $\boldsymbol{y}(n)$ sequence generates $\boldsymbol{y}_{t}(n)$ sequence. At the receiver side, removing CP symbols yields $\boldsymbol{y}_{r}(n)$ sequence that may be expressed as the cyclic convolution of $\boldsymbol{y}(n)$ and the impulse response of multipath fading channel. Employing FDE for $\boldsymbol{y}_{r}(n)$ yields $\boldsymbol{y}_{E q}(n)$, where FDE needs an $N$-point DFT to transfer symbols to frequency domain and an $\mathrm{N}$-point IDFT to return them

$$
\boldsymbol{x}_{t K}(n)+\frac{\boldsymbol{x}_{t K}^{e}(n)}{g_{K}(n)}
$$

Fig. 1 Extended-GFDM scheme 
back to time domain. Subsequently, $\boldsymbol{x}_{r i}^{e}(n)$ for $i=1: K$ are $\mathrm{N}$-symbol sequences obtained as

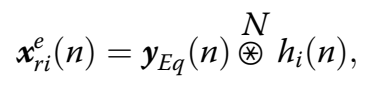

and then $K$-factor down-sampling operations yield $x_{r i}(n)$ symbols as

$$
x_{r i}(n)=x_{r i}^{e}(n K) \text { for } n=0: M-1 .
$$

Since each $g_{k}(n)$ is a band-passed filter located at a separate part of frequency domain, it can be considered as a subcarrier waveform. Assuming the sampling time to be $T_{\mathrm{s}}$, (3) shows that it will take $K T_{s}$ seconds to transmit one symbol of each subcarrier, and thus, $M$ symbols of each branch are carried within $M$ time slots taking the total duration of $M K T_{s}$ seconds. Therefore, the scheme is $K$-subcarrier and $M$ time slot modulation with block based output of $N=M K$ symbols. This result is in contrast with OFDM block (OFDM symbol) in which each subcarrier transmits one symbol during one time slot. The generic scheme gains some architectural advantages that are described as follows:

Low latency: Since equalization is processed within one block, extended-GFDM has the advantage of low delay. In addition, cyclic filtering used in extended-GFDM scheme prevents to generate tail symbols, and thus, it has lower latency compared to linear filtered multicarrier modulations.

Spectral efficiency: Extended-GFDM improves guard time overhead ratio compared with OFDM through sharing one $\mathrm{CP}$ among multiple time slots.

Flexible structure: In wireless cellular networks, small-

$M$, large- $K$ configurations can be employed for downlink to provide multiple access and large- $M$, small- $K$ configurations may be used for uplink to mitigate PAPR.

Assume $p(n)$ to be the impulse response of a prototype filter. Substituting $g_{i}(n)=p(n) \exp \left(j 2 \pi \frac{(i-1) n}{K}\right)$ for $i=1$ : $K$ in Fig. 1 creates a modulated filter bank; $y(n)$ is thus obtained as

$$
\boldsymbol{y}(n)=\sum_{i=1}^{K} \boldsymbol{x}_{t i}^{e}(n) \stackrel{N}{\circledast}\left(p(n) \exp \left(j 2 \pi \frac{(i-1) n}{K}\right)\right)
$$

In GFDM transmitter, data of $i$ branch is filtered by use of a prototype filter and shifted in the frequency domain through multiplying by $\exp \left(j 2 \pi \frac{(i-1) n}{K}\right)$ factor [13]; $y(n)$ is thus given as

$$
\boldsymbol{y}(n)=\sum_{i=1}^{K}\left(\boldsymbol{x}_{t i}^{e}(n) \stackrel{N}{\circledast} p(n)\right) \times \exp \left(j 2 \pi \frac{(i-1) n}{K}\right)
$$

It is straightforward to show (6) and (7) are equal, and therefore, employing $g_{i}(n)=p(n) \exp \left(j 2 \pi \frac{(i-1) n}{K}\right)$ in the extended-GFDM scheme leads to GFDM structure.

\section{Filter bank design for GOFDM transceiver}

GOFDM emerges through employing synthesis and analysis PR-QMF banks at the transmitter and receiver sides of extended-GFDM scheme, respectively. Since GOFDM follows extended-GFDM structure, it takes the common architectural advantages of extended-GFDM described in previous section. In this section, we will address the design of PR-QMF bank to achieve low OOB spectrum leak and low order of computational complexity when employed in the GOFDM structure.

\subsection{Background}

As seen in Fig. 2, PR-QMF bank consists of synthesis filter bank as multiplexing part and analysis filter bank as demultiplexing part. Connecting the output of the synthesis part directly to the input of the analysis part will completely reconstruct the original inputs of the filter bank. Furthermore, in PR-QMF bank, synthesis filters are matched to corresponding analysis filters [14]. In addition, perfect reconstruction property is preserved even when the convolutions are performed circularly, and thus, PR-QMF banks can also be classified as cyclic orthogonal filter banks [15]. Two-band PR-QMF banks are the simplest form of PRQMF banks which are easily designed by using a prototype filter. Assume $h(n)$ to be $L$-tap prototype filter-derived such that $L$ is an even number and its $Z$ transform satisfies the following condition:
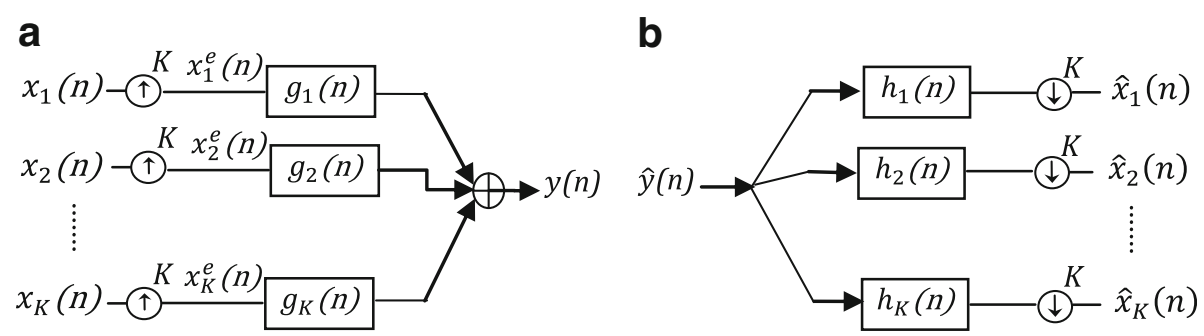

Fig. 2 Cyclic filter bank. a Synthesis part. b Analysis part 


$$
\begin{aligned}
& H(z) H\left(z^{-1}\right)+H(-z) H\left(-z^{-1}\right)=R(z)+R(-z) \\
& \quad=\text { Constant. }
\end{aligned}
$$

Perfect reconstruction property yields [16]

$$
\begin{aligned}
& h_{1}(n)=h(n), \\
& h_{2}(n)=(-1)^{(n+1)} h(L-1-n), \\
& g_{1}(n)=h(L-1-n),
\end{aligned}
$$

and

$$
g_{2}(n)=(-1)^{n} h(n)
$$

where $h_{1}(n)$ and $h_{2}(n)$ are low-pass and high-pass filters at the synthesis part and $g_{1}(n)$ and $g_{2}(n)$ are low-pass and high-pass filters at the analysis part of 2-band PRQMF bank, respectively. Based on (8), considering $R(z)$ $=H(z) H\left(z^{-1}\right)$, the $L$-tap prototype filter of 2-band PRQMF bank can be obtained as the causal part of $2 L$-tap half-band filter $R(z)$. The half-band filter $R(z)$ is a linear phase FIR filter, and thus, we may use the ParksMcClellan algorithm to derive $R(z)$ so as to provide the desired features for prototype filter $H(z)$ [17].

Using a uniform tree structure of $\log _{2} K$ levels, 2-band filter banks can be used for building $K$-band filter banks for the case where $K$ is a power of two. By using the same prototype filter for generating 2-band filter banks in all levels of the tree, we may obtain the conventional tree-structured filter bank. However, 2-band filter banks used in different levels of a tree, even at each node of the same tree level, can be different for the most general case. Figure 3 shows 4-band tree-structured filter bank in which the filters with superscripts 1 and 2 belong to the first and second levels of the tree, respectively.

\subsection{Optimized tree-structured PR-QMF bank}

In this part, we introduce the tree-structured PR-QMF filter bank with localized frequency response sub-band filters. Our approach is the design of non-conventional tree structure wherein different levels of tree have different 2-band filter banks but 2-band filter banks of the same level are similar. Let $M$ be the size of input sequences at the synthesis part of $K$-band tree-structured filter bank and $h^{i}(n)$ be the impulse response of the prototype filter employed to derive 2-band filter banks used in $i$ th level of the tree. The OOB spectrum leak depends on the behaviour of the filters within the passband and stopband. The optimization is thus aimed in designing $h^{i}(n)$ for $i=1: \log _{2} K$ such that the following optimization problem is satisfied

$$
\begin{array}{cl}
\min & E i \\
\text { Subject to } & E_{i}=\int_{f \notin \text { pasband }}\left|H^{i}(f)\right| d f \\
& R^{i}(z)+R^{i}(-z)=\text { Constant } \\
& R^{i}(z)=H^{i}(z) H^{i}\left(z^{-1}\right) \\
\text { variables } & H^{i}(f)
\end{array}
$$

where $H^{i}(f)$ and $H^{i}(z)$ are frequency response and $Z$ transform of $h^{i}(n)$, respectively. Substituting $h^{i}(n)$ for $h(n)$ in (9-12) yields $g_{1}^{i}(\mathrm{n}), g_{2}^{i}(\mathrm{n}), h_{1}^{i}(\mathrm{n})$ and $h_{2}^{i}(\mathrm{n})$ and, subsequently, the optimized $K$-band tree-structured filter will be established when the 2-band filter banks used in the $i$ th level of tree are obtained by use of the derived impulse responses $g_{1}^{i}(\mathrm{n}), g_{2}^{i}(\mathrm{n}), h_{1}^{i}(\mathrm{n})$ and $h_{2}^{i}(\mathrm{n})$ for $i=$ $1: \log _{2} K$.

In our approach, first, the Parks-McClellan algorithm is used to design $R^{i}(n)$ where the designing parameters are length, cut-off frequency and maximum ripple of the filter and then, $h^{i}(n)$ is derived as the causal part of $R^{i}(n)$. We determine the designing parameters of $R^{i}(n)$ so that $E_{i}$ is minimized for $h^{i}(n)$. Noting that going from each level to the successive higher one, the sequence size is duplicated, and hence, the size of input sequences at the $i$ th level of tree is $2^{i-1} M$ which is changed to $2^{i} M$ after up-sampling by the order of two. In order to preserve the size of filtered sequences through cyclic filtering, the filters length has to be less than or equal to the size of filtered sequences, and thus, we have

$$
L_{i} \leq 2^{i} M,
$$

where $L_{i}$ is the length of $h^{i}(n)$. Since more spread in the time domain permits designing of more localized sub-band filters in the frequency domain, the length of $h^{i}(n)$ is chosen to have the maximum possible value, i.e., $L_{i}=2^{i} M$. As a consequence, the order of respective half-band filter $R^{i}(n)$ will be $2 \times 2^{i} M-1$. In addition, cut-off frequency is set at the middle of frequency space (i.e., 0.5 for normalized frequency space). $R^{i}(n)$ is then derived by use of Parks-McClellan algorithm and on the conditions that the order and cut-off frequency are fixed and the maximum ripple is numerically determined such that the achieved $h^{i}(n)$ minimizes $E_{i}$. Figure 4 compares frequency response of 32-tap optimum prototype filter (case 2) versus two non-optimum 32-tap prototype filters of cases 1 and 3 with larger and smaller maximum-ripples, respectively. The filters are derived as the causal parts of 64-tap half-band filters with the order and cut-off frequency equal to 61 and 0.5 , respectively. For case 2, the maximum-ripple of respective half-band filter is numerically derived as 0.0121 while the maximum-ripples of cases 1 and 3 are chosen as 0.1 and 0.001 , respectively. The values of $E_{i}$ for cases 1,2 , and 3 are $0.0944,0.064$, and 0.0712 , respectively. The results show optimum filter with lowest $E_{i}$ value may have larger 

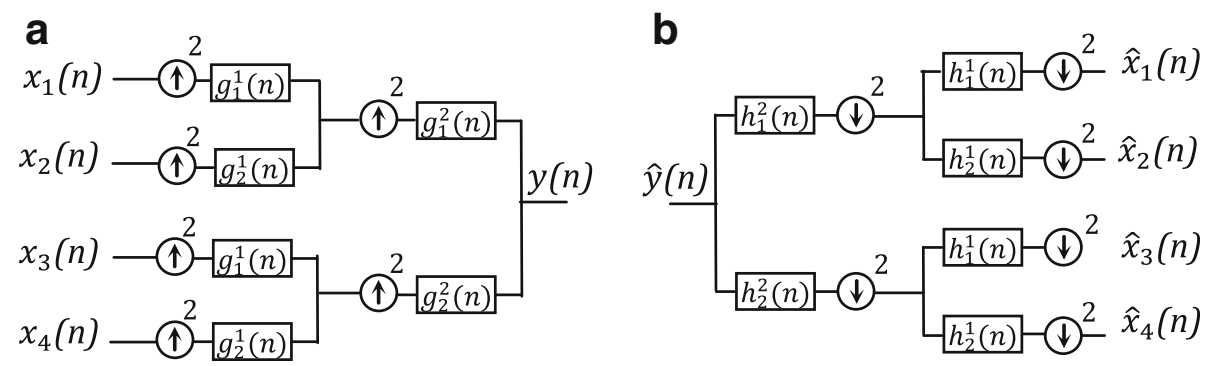

Fig. 3 Four-band tree-structured filter bank. a Synthesis part. b Analysis part

maximum-ripple compared to non-optimum prototype filters.

\subsection{Low-complexity tree-structured PR-QMF bank}

In this section, we propose a practical implementation algorithm in order to reduce the computational complexity of the optimum cyclic tree-structured filter bank. In order to reach this goal, the cyclic tree-structured filter bank is implemented in the frequency domain using Discrete Fourier Transform (DFT) and Inverse DFT (IDFT), where $M$-point DFT and IDFT are defined as:

$$
\begin{aligned}
& X(k)=\frac{1}{\sqrt{M}} \sum_{n=0}^{M-1} x(n) W_{M}^{k n} \text { for } k=0: M-1 \\
& x(n)=\frac{1}{\sqrt{M}} \sum_{k=0}^{M-1} X(k) W_{M}^{-k n} \text { for } n=0: M-1,
\end{aligned}
$$

where $W_{M}=e^{-j\left(\frac{2 \pi}{M}\right)}$. Time domain operations for a 2band cyclic filter bank are cyclic convolution, upsampling, and down-sampling by the order of two. Throughout the paper, we will denote the frequency domain operations equivalent to the time domain factor 2 up-sampling and down-sampling as 2-Copy and 2-Fold, respectively, where the definitions of 2-Copy and 2-Fold are derived in the following lemmas.

Lemma 1 If the M-symbol sequence $\boldsymbol{x}(n)$ is up-sampled by the factor of 2 to generate $\boldsymbol{x}^{e}(n)$, then $\boldsymbol{X}^{e}(k)=2$-Copy $\{\boldsymbol{X}(k)\}$, where $\boldsymbol{X}(k)$ is a sequence of M-point DFT coefficients of $x(n)$, $X^{e}(k)$ is a sequence of 2M-point DFT coefficients of $\boldsymbol{x}^{e}(n)$ and $X^{\mathrm{e}}(k)=2-\operatorname{Copy}\{X(k)\}=\left\{\begin{array}{l}\frac{1}{\sqrt{2}} X(k) \text { for } k=0: M-1 \\ \frac{1}{\sqrt{2}} X(k-M) \text { for } k=M: 2 M-1\end{array}\right.$

Lemma 2 If $2 M$-symbol sequence $\boldsymbol{x}(n)$ is down-sampled by the factor of 2 to generate $x^{d}(n)$, then $X^{d}(k)=2$-Fold $\{X(k)\}$, where $X(k)$ is a sequence of 2M-point DFT coefficients of $\boldsymbol{x}(n)$ and $X^{d}(k)$ is a sequence of M-point DFT coefficients of $x^{d}(n)$ and 2-Fold $\{X(k)\}=\frac{1}{\sqrt{2}}[X(k)+X(k+M)]$ for $k=0,1, \ldots, M$.

Proof In the earlier study, we have expressed up- and down-sampling by the factor $K$ in the frequency domain by use of DFT coefficients [11]. Substituting $K$ with 2 in the derived results yields the results of Lemmas 1 and 2 .

Further, we know that cyclic convolution of the $M$ symbol sequence $\boldsymbol{x}(n)$ with the $M$-coefficient filter $g(n)$ is equivalent to the multiplication of DFT coefficients of $\boldsymbol{x}(n)$ and frequency domain coefficients of $g(n)$ given by

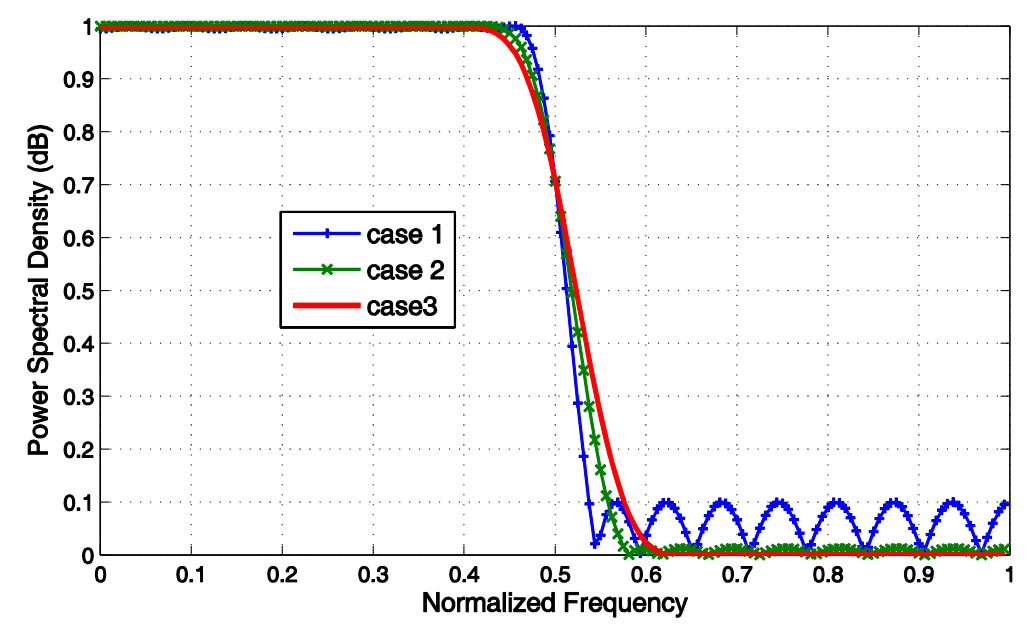

Fig. 4 Frequency responses of 32-tap prototype filters 


$$
G(k)=\sum_{n=0}^{M-1} g(n) W_{M}^{k n} \text { for } k=0: M-1
$$

Substituting up-sampling, down-sampling, and cyclic convolution operations of $K$-band tree-structured filter bank with 2-Copy, 2-Fold, and multiplication operations yields a new structure that we call it as frequency domain tree structure (FDTS) filter bank. Consequently, in order to completely implement the filter bank in the frequency domain, at the first stage of synthesis part, a set of $K$ blocks of $M$-point DFT are required to transfer input blocks to the frequency domain. The $K$-band FDTS synthesis filter bank is the subsequent stage, and then, $N$-point IDFT is required to return these symbols from the frequency domain back to the time domain. At the analysis part, the first stage is an $N$-point DFT. The $K$-band FDTS analysis filter bank and $K$ blocks of $M$-point DFT are the subsequent stages.

Figure 5 shows how to implement a 4-band treestructured filter bank in frequency domain where $\boldsymbol{x}_{i}(n)$ and $\hat{\boldsymbol{x}}_{i}(n)$ for $i=1: 4$ are $M$-symbol sequences and $\mathbf{y}(n)$ and $\hat{\mathbf{y}}(n)$ are $4 M$-symbol sequences, respectively. In general case, based on the results of the previous section derived for optimum filter bank, when the inputs at the synthesis part are $M$-symbol sequences, $g_{1}^{i}(\mathrm{n}), g_{2}^{i}(\mathrm{n}), h_{1}^{i}$ (n) and $h_{2}^{i}(\mathrm{n})$ are $2^{i} M$-coefficient filters. Substituting $g_{1}^{i}$ $(\mathrm{n}), g_{2}^{i}(\mathrm{n}), h_{1}^{i}(\mathrm{n})$ and $h_{2}^{i}(\mathrm{n})$ for $g(n)$ in (17) yields the frequency domain coefficients of the filters to be employed at the $i$ th level of FDTS filter bank, i.e., $G_{1}^{i}(k), G_{2}^{i}(k), H_{1}^{i}$ $(k)$ and $H_{2}^{i}(k)$. Finally, it should be noted that since cyclic filtering creates a clockwise circular shift, outputs of the analysis part of PR-QMF bank are not exactly equal to the corresponding inputs of synthesis part. In order to compensate such shifts and obtain full reconstruction cyclic filter bank, it is enough to change frequency domain coefficients of the synthesis filters for $i=1: \log _{2} K$ as

$$
\begin{aligned}
& \hat{G}_{1}^{i}(k)=G_{1}^{i}(k) W_{2^{i} M}^{2^{i} M(k-1)} \text { for } k=0: 2^{i} M-1 \\
& \hat{G}_{2}^{i}(k)=G_{2}^{i}(k) W_{2^{i} M}^{2^{i} M(k-1)} \text { for } k=0: 2^{i} M-1
\end{aligned}
$$

\subsection{Complexity analysis}

In this part, the complexities of frequency and time domain implementations of tree-structured filter bank are compared. The complexity is calculated in terms of the number of needed basic operations of additions and multiplications to generate one output block. The filter bank is the optimal $K$-band tree-structured filter bank where the inputs at the synthesis part are sequences of $M$ symbols. In general, the coefficients of the filters may be complex numbers, and so, all multiplications and additions are generally complex instructions. We assume that the filter coefficients and the DFT coefficients of the filters are known at the receiver side and they are registered in the buffer in the initialization step.

\subsubsection{Frequency domain implementation}

Figure 6a, b shows a typical 2-band frequency domain synthesis and analysis filter banks used at the $i$ th level of the synthesis and analysis parts of optimum FDTS filter bank, respectively. Let $V_{1}(k), V_{2}(k)$ be $2^{i-1} M$-symbol input sequences of 2-band synthesis filter bank. Based on Lemma 1 , the next 2-Copy operations generate $2^{i} M$-symbol sequences whose symbols are given as

$$
\begin{aligned}
& \underset{V}{1 e}(k)=V_{1}^{e}\left(k+2^{i-1} M\right)=V_{1}(k) \text { for } k=0: 2^{i-1} M-1 \\
& { }_{V}^{2 e}(k)=V_{2}^{e}\left(k+2^{i-1} M\right)=V_{2}(k) \text { for } k=0: 2^{i-1} M-1,
\end{aligned}
$$

and, subsequently, the output symbols of synthesis part are obtained as
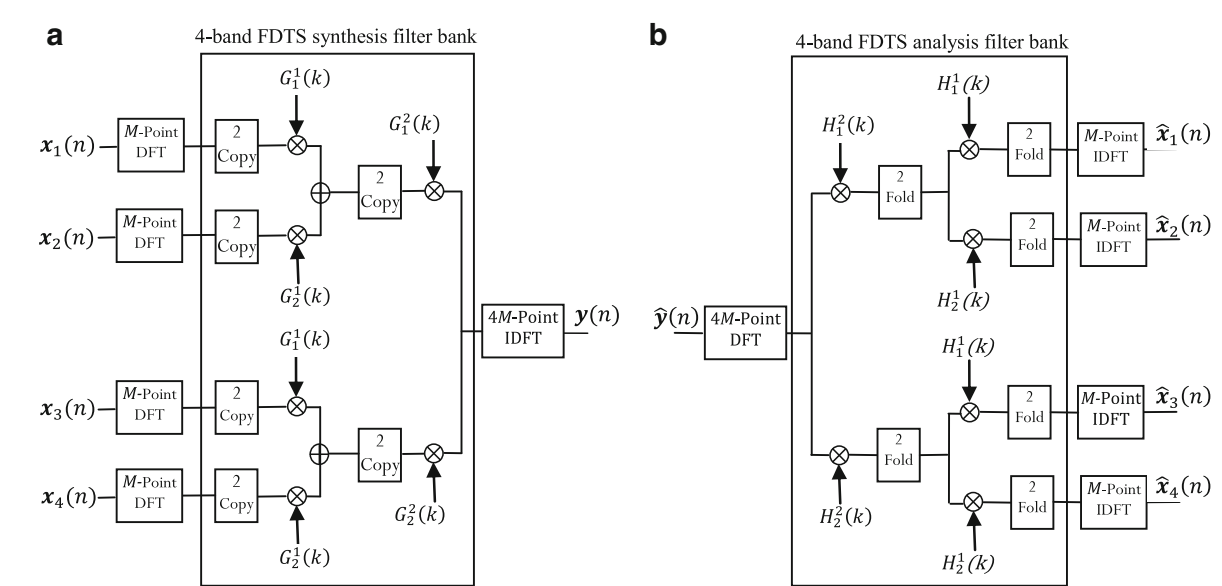

Fig. 5 Frequency domain implementation of 4-band tree-structured filter bank. a Synthesis part. $\mathbf{b}$ Analysis part 


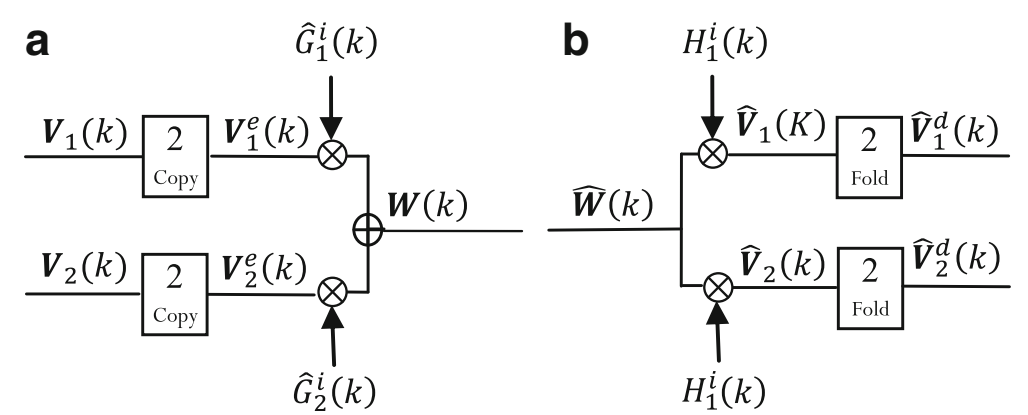

Fig. 6 Two-band $\mathbf{a}$ synthesis and $\mathbf{b}$ analysis filter bank of ith level of FDTS filter bank

$$
W(k)=V_{1}^{e}(k) \times \hat{G}_{1}^{i}(k)+V_{2}^{e}(k) \times \hat{G}_{2}^{i}(k) \text { for } k=0: 2^{i} M-1
$$

For 2-band analysis filter bank, letting $\hat{W}(k)$ be $2^{i} M$ symbol input sequence yields

$$
\begin{aligned}
& \hat{V}_{1}(k)=\hat{W}(k) \times H_{1}^{i}(k) \text { for } k=0: 2^{i} M-1 \\
& \hat{V}_{2}(k)=\hat{W}(k) \times H_{2}^{i}(k) \text { for } k=0: 2^{i} M-1
\end{aligned}
$$

subsequently, based on Lemma 2, output symbols of 2band analysis filter bank are obtained as

$$
\begin{aligned}
& \hat{V}^{1 d}(k)=\frac{1}{2}\left[\hat{V}_{1}(k)+\hat{V}_{1}\left(k+2^{i-1} M\right)\right] \text { for } k=0: 2^{i-1} M-1 \\
& \hat{V}^{2 d}(k)=\frac{1}{2}\left[\hat{V}_{2}(k)+\hat{V}_{2}\left(k+2^{i-1} M\right)\right] \text { for } k=0: 2^{i-1} M-1
\end{aligned}
$$

Based on (22), the 2-band frequency domain synthesis filter banks used at the $i$ th level of FDTS synthesis filter bank require $2 \times 2^{i} M$ multiplications and $2^{i} M$ additions. The $K$-band FDTS synthesis filter bank consists of $\log _{2} K$ levels, and there are $\frac{K}{2^{i}} 2$-band filter banks at the $i$ th level. Therefore, the number of multiplications and additions performed in the synthesis part of FDTS is

$$
\left\{\begin{array}{l}
\sum_{i=1}^{\log _{2} K} \frac{K}{2^{i}} \times 2 \times 2^{i} M=2 K M \log _{2} K \\
\sum_{i=1}^{\log _{2} K} \frac{K}{2^{i}} \times 2^{i} M=K M \log _{2} K
\end{array}\right.
$$

mults adds.

In order to implement $K$-band synthesis filter bank in the frequency domain, the total basic operations needed consists of basic operations for the calculation of $K$ blocks of $M$-point DFT, $K$-band FDTS, and $N$-point IDFT. $K$ is always a power of two and $N=M K$, thus assuming that $M$ to be a power of two yields $N$ to be also a power of two. Therefore based on the radix-2 FFT algorithm, the overall number of basic operations is obtained as

$$
\begin{cases}K \frac{M}{2} \log _{2} M+2 K M \log _{2} K+\frac{N}{2} \log _{2} N & \text { mults } \\ K M \log _{2} M+K M \log _{2} K+N \log _{2} N & \text { adds. }\end{cases}
$$

On the other hand, referring to Eqs. (23-26), 2-band analysis filter banks used at $i$ th level of analysis part need $2 \times$ $2^{i} M$ multiplications for two multiplication operators and $2 \times$ $2^{i-1} M$ additions for two 2 -Fold operators. Therefore, the 2band analysis and synthesis filter banks used at the $i$ th level of FDTS filter bank need equal number of basic mathematical calculations, and consequently, the total number of basic mathematical operations of synthesis and analysis parts of frequency domain implementation are similar.

\subsubsection{Time domain implementation}

The inputs of the $i$ th level of synthesis part are blocks of $2^{i-1} M$ symbols, and thus, after up-sampling, the size of input blocks is changed to $2^{i} M$ where half of them are zeroes. Two-band filter banks used at the $i$ th level of analysis part require two $2^{i} M$-point cyclic convolutions and one $2^{i} M$-point addition at the end. Therefore, number of basic operations performed for the 2-band synthesis filter banks used at $i$ th level of synthesis part is

$$
\begin{cases}2^{2 i} M^{2} & \text { mults } \\ 2^{i} M\left(2^{i} M-1\right) & \text { adds. }\end{cases}
$$

The tree is built of $\log _{2} K$ levels where the $i$ th level at the synthesis part consists of $\frac{K}{2^{i}}$ 2-band synthesis filter bank. Therefore, the total number of basic operations of the synthesis part yields as

$$
\begin{cases}\sum_{i=1}^{\log _{2} K} \frac{K}{2^{i}} \times 2^{2 i} \quad M^{2}=N M \sum_{i=1}^{\log _{2} K} 2^{i} & \text { mults } \\ \sum_{i=1}^{\log _{2} K} \frac{K}{2^{i}} \times 2^{i} M\left(2^{i} M-1\right)=N \sum_{i=1}^{\log _{2} K}\left(2^{i} M-1\right) & \text { adds. }\end{cases}
$$

In a similar approach, it can be shown that the numbers of basic operations at the analysis part is equal to 
Table 1 Computational complexity of optimum tree-structured analysis/synthesis filter bank

\begin{tabular}{lll}
\hline & $\begin{array}{l}\text { Total number } \\
\text { of multiplications }\end{array}$ & $\begin{array}{l}\text { Total number } \\
\text { of additions }\end{array}$ \\
\hline Frequency domain implementation & $\frac{N}{2} \log _{2} M+2 N \log _{2} K+\frac{N}{2} \log _{2} N$ & $N \log _{2} M+N \log _{2} K+N \log _{2} N$ \\
Time domain implementation & $2 N^{2}-2 N M$ & $2 N^{2}-2 N M-\frac{N \log _{2} K}{2}\left(\log _{2} K+1\right)$ \\
\hline
\end{tabular}

those of the synthesis part. The results are presented in Table 1. It is seen that the complexity of frequency and time domain implementations are of the order of $N \log _{2} N$ and $N^{2}$, respectively.

\subsection{GOFDM system model based on optimum low- complex filter bank}

Deploying the optimum low-complexity treestructured PR-QMF bank in the generic scheme of Fig. 1 yields the block diagram of Fig. 7 wherein lower case letters denote time domain symbols and higher case letters denote frequency domain symbols. As stated before, the advantage of implementing treestructured PR-QMF filter bank in frequency domain instead of time domain is reducing the computational complexity of the structure. This advantage is more highlighted at the receiver side wherein the existing FDE implies that the received symbols are transferred to the frequency domain even in case of time domain implementation.

\section{Simulation results}

\subsection{Simulation setup}

In what follows, we compare GOFDM with GFDM in terms of spectrum localization, error performance, numerical complexity, and peak to average power ratio. Optimum tree-structured PR-QMF bank is employed for GOFDM. In the GFDM structure, the prototype filter is root-raised cosine (RRC) filter with roll-off factor of 0.3. Setting up the configuration parameters of filter banks needs some consideration. Provided that $M$ and $K$ being even numbers GFDM transmit matrix becomes singular [13] but PR-QMF bank does not face such a problem and we are permitted to use $M$ and $K$ both as a power of 2 . In the simulations, we evaluate two configurations where the number of subcarriers in GFDM and GOFDM schemes for configuration cases 1 and 2 are 256 and
16, respectively. Therefore, we encounter the GFDM limitation of using odd number for $M$ values. Hence, $M$ is chosen as 9 and 129 for GFDM scheme and the nearest power 2 numbers, i.e., 8 and 128 are chosen for GOFDM scheme in the configuration cases 1 and 2, respectively. In two GOFDM configurations, $M \times K$ $=2048$ and the number of CP symbols is equal to 64; thus, both configurations have similar spectral efficiency. When OOB emission is analyzed, configuration with large number of subcarriers, i.e., case 1 is taken into account while PAPR is analyzed for configuration with small number of subcarriers, i.e., case 2 . Both configurations are evaluated in terms of error performance and numerical complexity. The channel profile and other simulation parameters are presented in Table 2.

\subsection{OOB leakage}

In order to reduce $\mathrm{OOB}$ emission, the normal approach is to use empty subcarriers at the corner sides of assigned bandwidth. For block-based modulations, abrupt changes of signal between successive blocks result in high $\mathrm{OOB}$ emission. Windowing is thus exploited at the transmitter side by multiplying output blocks with a window function to smooth abrupt changes and combat the $\mathrm{OOB}$ emission. For the case of windowed waveforms in addition to $\mathrm{CP}$, cyclic suffix (CS) is also inserted. Through the simulations, G prefix denotes windowed waveform and GW prefix denotes windowing and guard subcarriers are simultaneously applied for a waveform. According to LTE standard for the case of 256 subcarriers, 150 subcarriers are occupied as allocated bandwidth. We consider six empty subcarriers of each side as guard band and, consequently, power spectral densities (PSDs) leak to other empty subcarriers may be considered as OOB emission. For the case of GOFDM, despite what might be expected, frequency responses of sub-band filters of the $K$-band tree-structured filter

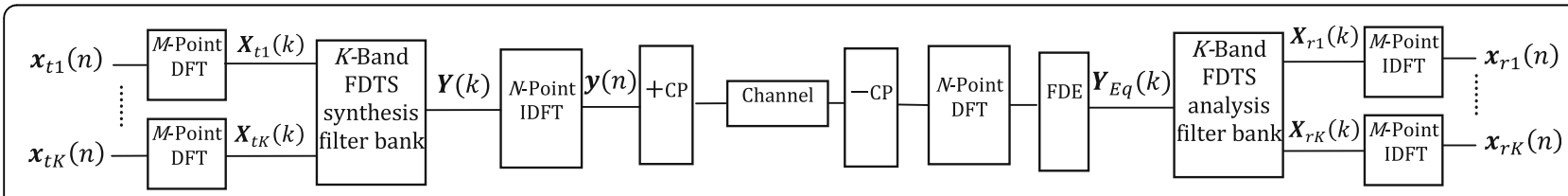

Fig. 7 GOFDM block diagram based on optimum low-complexity tree-structured PR-QMF bank 
Table 2 Simulation parameters

\begin{tabular}{|c|c|c|c|}
\hline Parameters & GFDM & GOFDM & OFDM \\
\hline \multicolumn{4}{|l|}{ General parameters } \\
\hline Number of CP symbols $\left(N_{c p}\right)$ & 64 & 64 & 64 \\
\hline Number of CS symbols $\left(N_{C S}\right)$ & 32 & 32 & 16 \\
\hline Gaussian window & 32 samples in both sides & & $\begin{array}{l}16 \text { samples in } \\
\text { both sides }\end{array}$ \\
\hline $\begin{array}{l}\text { Channel impulse response } \\
\vec{C}=\left[C_{0} \ldots C_{N_{c p}-1}\right]\end{array}$ & $\begin{array}{l}c_{i} \text { is complex Gaussian random variable } \\
\text { with zero mean and variance of } e^{-i / 10}\end{array}$ & & \\
\hline Filter bank & RRC modulated filter bank roll-off $=0.3$ & $\begin{array}{l}\text { Optimum tree-structured } \\
\text { PR-QMF bank }\end{array}$ & Not used \\
\hline \multicolumn{4}{|l|}{ Configuration case 1} \\
\hline Number of subcarriers ( $K$ ) & 256 & 256 & 256 \\
\hline Number of time slots $(M)$ & 9 & 8 & 1 \\
\hline \multicolumn{4}{|l|}{ Configuration case 2} \\
\hline Number of subcarriers $(K)$ & 16 & 16 & 256 \\
\hline Number of time slots $(M)$ & 129 & 128 & 1 \\
\hline Mapping & 16-QAM & 16-QAM & 16-QAM \\
\hline
\end{tabular}

bank are disorderly arranged in the spectrum for the case where $K$ is higher than 2. The 4-band frequency split of the spectrum is illustrated in Fig. 8 for ideal band-pass filters. As explained, 4-band tree-structured filter bank has two stages, stage 1 consists of two 2band filter banks and stage 2 consists of one 2-band filter bank. It is seen in Fig. 8 that filter bank of stage 2 reverses the arrangement of two higher sub-bands and the frequency response of sub-band 3 comes before sub-band 4 . Therefore, the order of 4-band tree-structured filter bank in the spectrum may be shown as $\{1,2,4,3\}$. Similarly, 8 band tree-structured filter bank may be considered as a stage of two 4-band tree-structured filter banks prior to one 2-band filter bank. Combining two 4-band treestructured filter banks using next 2-band filter bank reverses the order of higher 4-band filter bank, and thus, the sub-bands are ordered in the spectrum as $\{1,2,4,3,7,8,6,5\}$.
Following such approach, one can find the order of subband filters of $K$-band tree-structured filter bank in the spectrum is matched to a sequence of gray numbers. In our case study, the 256-number gray sequence starting from 1 shows the order of subcarriers in the spectrum.

Figure 9 compares PSDs of OFDM, GFDM, and GOFDM. In order to evaluate OOB emission, OOB radiation parameter is defined as the ratio of the energy that is emitted into the $\mathrm{OOB}$ range and the amount of energy within the allocated bandwidth, i.e.,

$$
\text { OOB radiation }=\frac{|\mathrm{BW}|}{|\mathrm{OOB}|} \cdot \frac{\int_{f \in \mathrm{OOB}} P(f) d f}{\int_{f \in \mathrm{BW}} P(f) d f}
$$

where $B W$ and $O O B$ are the set of frequencies that are considered as in-band and out of band, respectively. The OOB radiations of G-OFDM, G-GFDM,

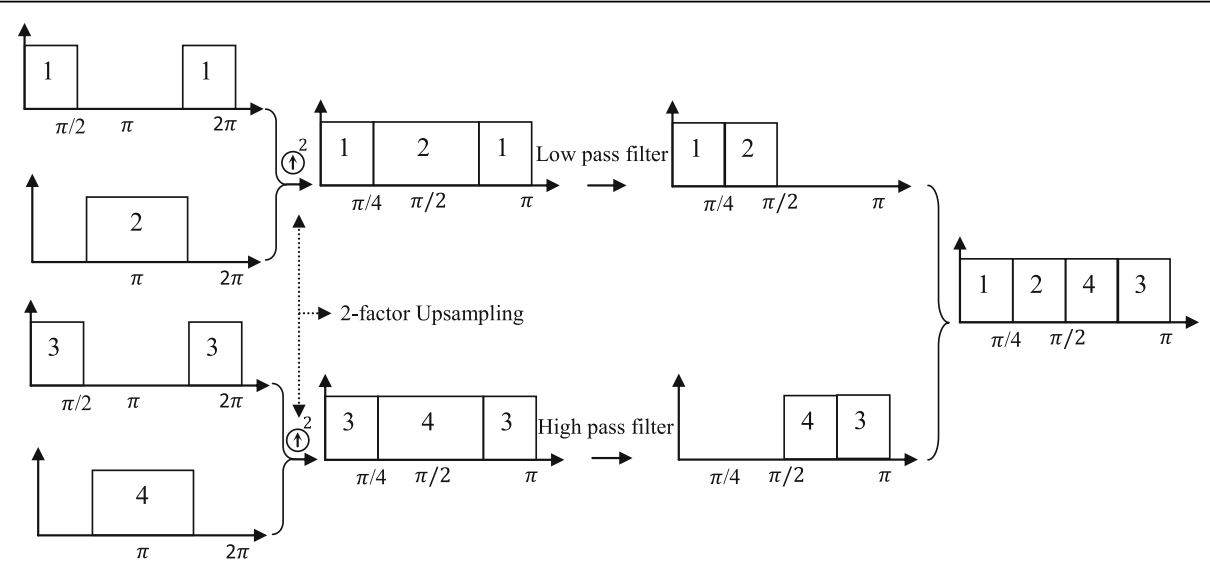

Fig. 8 The frequency split of spectrum in 4-band synthesis tree-structured filter bank of ideal filters 


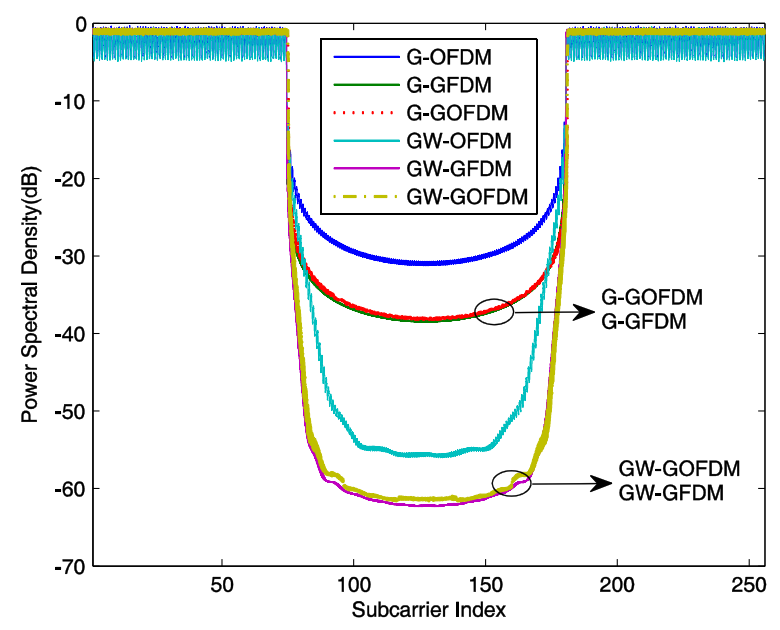

Fig. 9 Power spectral density comparison for 16-QAM

and G-GOFDM are calculated as $-25.7,-34.2$, and - $33.9 \mathrm{~dB}$, respectively. In case of applying both windowing and guard subcarriers, the OOB radiations of GW-OFDM, GW-GFDM, and GW-GOFDM are reduced to $-35.6,-50.5$, and $-49.8 \mathrm{~dB}$, respectively. As expected, the OOB emissions of cyclic multicarrier modulations are significantly less than that of OFDM. Furthermore, the optimized PR-QMF bank causes GOFDM OOB radiations to be only 0.3 and $0.7 \mathrm{~dB}$ more than that of GFDM for the cases of inserting guard subcarriers and employing window and inserting guard subcarriers together, respectively. We now compare the bandwidth efficiency for three modulations. Noting that the overhead symbols are CP and CS guard symbols in the time domain and empty guard subcarriers in the frequency domain, we define the spectral efficiency as the ratio of useful symbols to the total transmitted symbols during each output block, and then, the spectral efficiency of OFDM, GOFDM, and GFDM are $\frac{256}{256+64+16}=0.76, \frac{256 \times 9}{256 \times 9+64+32}=0.96$ and $\frac{256 \times 8}{256 \times 8+64+32}=0.95$, respectively. The results show that GOFDM transmitter yields almost the same level of OOB leakage and spectral efficiency as those of GFDM transmitter.

\subsection{Error performance}

In the case of GFDM MMSE receiver, an inverse matrix has to be calculated according to changes in noise power. Therefore, GFDM MMSE receiver suffers from high complexity, and DSIC/MF receiver is suggested to use instead. In the earlier study, we have proved, on the assumption of perfect synchronization, GOFDM MMSE receiver which simply consists of one-tap MMSE-FDE and analysis PR-QMF bank is the best GOFDM linear receiver in terms of error performance
[12]. On the other hand, GFDM DSIC/MF receiver is the combination of ZF-FDE, matched filters and DSIC algorithm. One-tap coefficients of MMSE-FDE and ZF-FDE for $k=1: N$ are defined as $\frac{1}{C(k)}$ and $\frac{C^{*}(k)}{|C(k)|^{2}+\sigma_{w}^{2}}$, respectively, where $C(k)$ and $\sigma_{w}^{2}$ are $N$-point DFT coefficients of channel impulse response and the power of white Gaussian noise, respectively.

In this study, OFDM, GFDM DSIC/MF with four iterations of DISC algorithm, and GFDM MMSE and GOFDM MMSE receivers are compared in terms of error performance for transmitting windowed signals through multipath fading channel when complete synchronization is assumed. At the first step, uncoded BER is analyzed. As seen in Fig. 10a for the case of configuration 1, while GOFDM MMSE slightly outperforms OFDM, the BER curves of GFDM MMSE and DSIC/MF receivers are tightly close to that OFDM. For the configuration case 2 shown in Fig. 10b, GOFDM and GFDM MMSE receivers outperform OFDM in terms of error performance for signal to noise ratio (SNR) larger than 12 and $15 \mathrm{~dB}$, respectively, and the situations are reversed for SNR smaller than these thresholds. However, there is a considerable gap between GFDM DSIC/MF and OFDM error performance curves which means the negative effect of self-interference at the receiver side of GFDM MF receiver is more serious for configuration case 2 compared with configuration case 1 . At the second step, the parallel concatenated convolutional code (PC-CC) with code rate $R=1 / 3$ is employed for transmitter side, and a soft demapper in combination with a turbo decoder with 10 iterations is employed at the receiver side. It is known that the performance of employed advanced receiver depends on the mean square error in the received symbols. Therefore, as seen in Fig. 10c, d, while GOFDM MMSE outperforms 

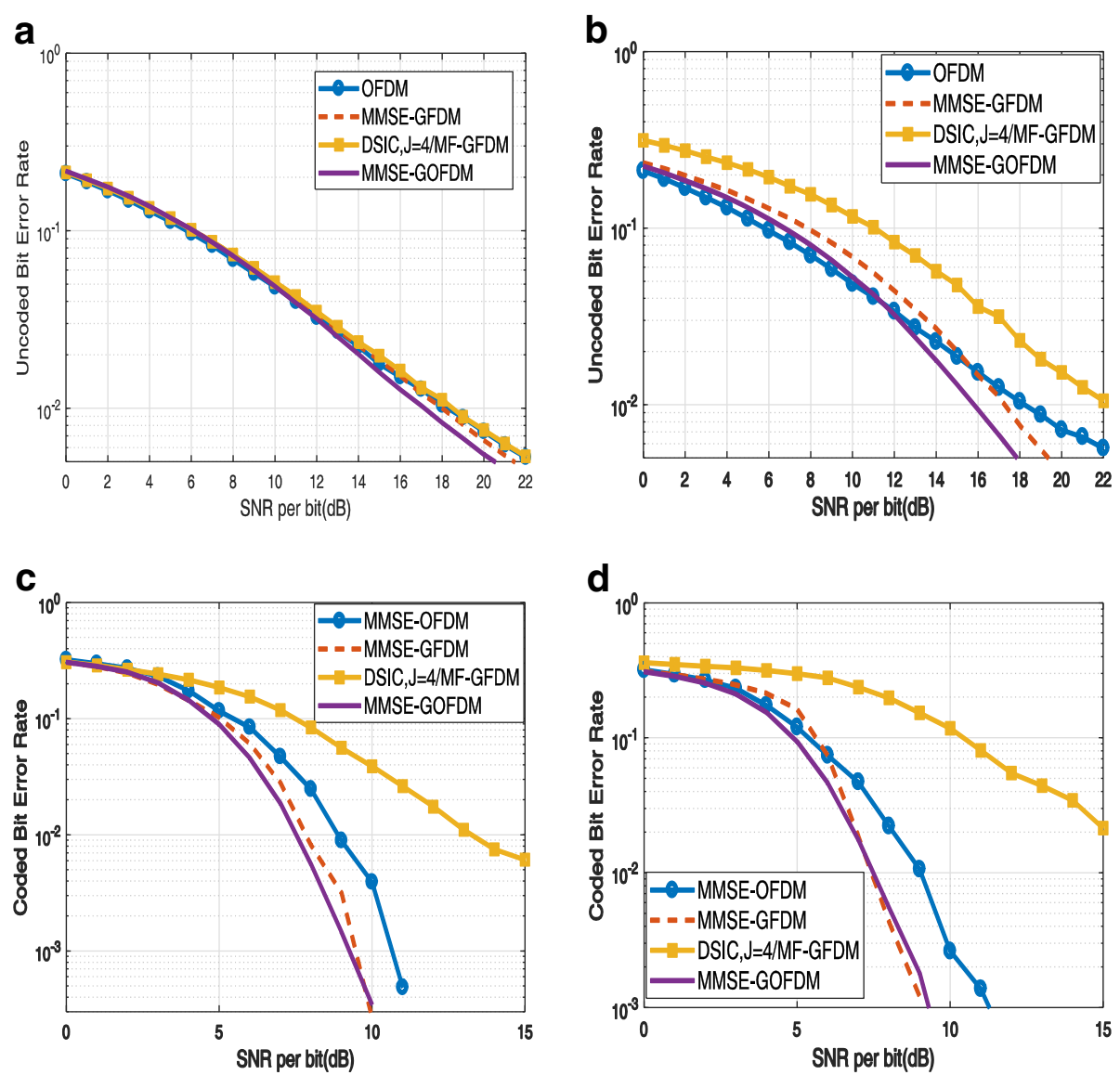

Fig. 10 Comparison of BER in multipath fading channel for 16-QAM where configuration is case $1(\mathbf{a}, \mathbf{c})$ and case 2 (b, d)

OFDM, there are significant gaps between coded BER curves of DSIC/MF GFDM and OFDM in both configurations. On the other hand, the coded error performance of GOFDM MMSE receiver compared to GFDM MMSE receiver depends on the configuration of filter bank and SNR value. It can be verified that BER curve of GOFDM MMSE is slightly lower/higher than that of GFDM MMSE for SNR smaller/larger than 9.7 and $7.5 \mathrm{~dB}$ for the configuration cases 1 and 2, respectively.

\subsection{Computational complexity}

Among the methods introduced to implement GFDM receivers, authors in [18] proposed the lowest complexity modem for GFDM zero-forcing and matched filter receivers. However, the proposed method does not support DSIC/MF receiver for the case of multipath fading channel. Therefore, sparse frequency domain processing method introduced in [19] still leads to lowest complexity for our case study. Based on this method, the number of multiplications in GFDM DSIC/MF receiver with the configuration of $K$ subcarriers and $M$ time slots ignoring the equalizer complexity is reduced to

$$
N\left[(2 J+1) \log _{2} M+(J+I)+\log _{2} N\right],
$$

where $I$ is the number of adjacent sub-bands wherein the frequency response of prototype filter has significant values and $J$ is the number of iterations for DSIC algorithm. $I$ depends on the frequency response of prototype filter and usually is small; however, $J$ depends on the order of QAM constellation and for the case of large QAM constellation, large $J$ is required to achieve good error performance [19].

Ignoring the equalizer part, the complexity of GOFDM receiver with the configuration of $K$ subcarriers and $M$ time slots is equivalent to the complexity of $K$-band analysis filter bank with an input block of $N=M K$ symbols. When $M$ and $K$ are a power of two, numerical complexity for such configuration is derived in (28).

We compare numerical complexity of GFDM DSIC/ MF and GOFDM MMSE receivers in terms of the number of multiplications. In the case of GOFDM, $K$ and $M$ are power of 2 numbers where $K$ is ranged from 16 to 2048 and $M$ is ranged from 4 to 128 . For the case of GFDM, $K$ has the same range but $M$ values increased by 1, i.e., $M=5,9,17,33,65,129$. The values of $M$ in 


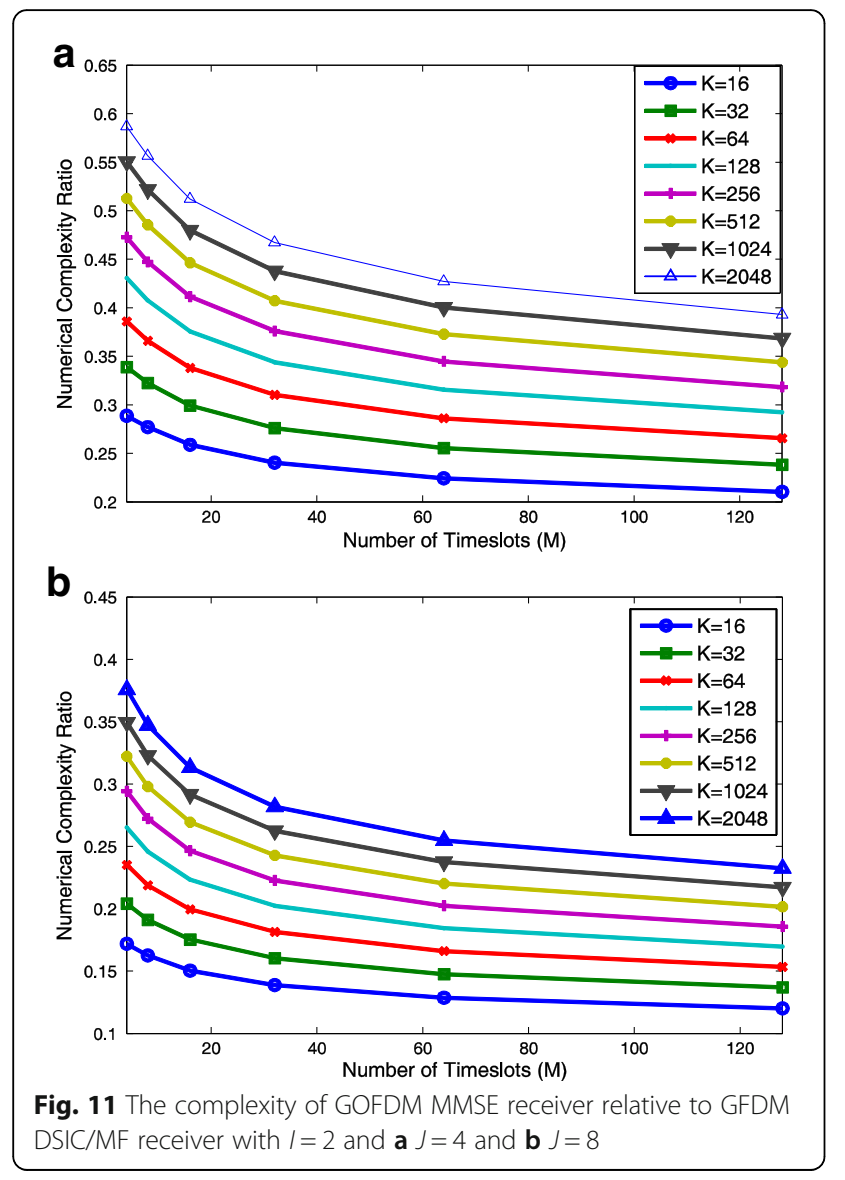

GFDM and GOFDM configurations are not equal; thus, we consider GOFDM configuration parameters as the reference and calculate its complexity relative to GFDM complexity with equal $K$ but $M$ values increased by 1 . As seen in Fig. 11a, b, GOFDM MMSE receiver achieves less numerical complexity compared to GFDM DSIC/ MF receiver especially for the case of large $M$ and large $J$ values. For example, in configuration case 1, GOFDM to GFDM complexity ratio for $J=4$ and $J=8$ are 0.4474 and 0.2724 , respectively, and in configuration case 2 , this parameter equals 0.2106 and 0.1203 for $J=4$ and $J=8$, respectively.

\subsection{PAPR}

The PAPR of $\boldsymbol{y}_{t}(n)$ sequence is defined as

$$
\operatorname{PAPR}\left(\boldsymbol{y}_{t}(n)\right)=\frac{\max \left(\left|y_{t}(n)\right|^{2}\right)}{\operatorname{average}\left(\left|y_{t}(n)\right|^{2}\right)}
$$

Larger PAPR values demand a higher linear range amplifier which is a challenge for designing low-cost terminals. Complementary cumulative density function (CCDF) of PAPR, i.e., the probability that the PAPR exceeds a certain value, is the typical measure to compare different systems in terms of PAPR. PAPR value of multicarrier schemes depends on the number of subcarriers, and the schemes with a few number of subcarriers perform better than the schemes with large number of subcarriers [20] in terms of PAPR. Authors in [21] showed considering GFDM multicarrier scheme for the uplink of a cellular network wherein only one subcarrier assigned to each user yields smaller PAPR compared to OFDM scheme. Therefore, we consider GFDM and GOFDM with the configuration case 2 for an uplink scenario where one subcarrier assigned to each of 16th users. For the case of OFDM, 16 contiguous subcarriers are allocated to each user. As the benchmark, single-carrier FDM system is considered. Then, a pulse-shaping raised cosine filter with roll-off $=0.5$ is applied in all schemes. The average values of CCDF of PAPR of transmit signals of the waveforms are shown in Fig. 12. It is seen, OFDM is significantly outperformed by GFDM and GOFDM in terms of PAPR. It can also be verified that GOFDM is superior to single-carrier FDM but it is outperformed by GFDM in terms of PAPR. According to results of previous parts, GFDM transmitter achieves such superiority at the costs of more computational complexity or lower error performance of the receiver side.

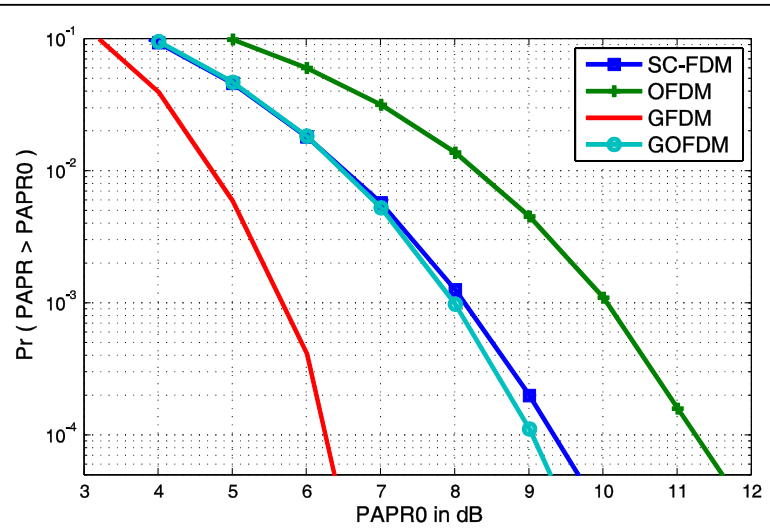

Fig. 12 PAPR comparison for 16-QAM constellation 


\section{Conclusions}

GOFDM encompasses the architectural advantages of GFDM including high spectral efficiency, low latency, and flexible structure. The novelty of current work relies on exploiting a fast and optimized PR-QMF bank to be employed in the GOFDM structure. As a consequence, GOFDM transmitter yields the same level of OOB leakage as that of GFDM transmitter and GOFDM receiver yields lower computational complexity compared with GFDM DSIC/MF receiver. Further, it was shown that GOFDM MMSE receiver is superior to OFDM in terms of error performance when modern channel coding is used in the setup. In the similar conditions, GFDM DSIC/MF receiver could not perform adequately, and thus, it was required to use high complexity MMSE receiver for GFDM to achieve acceptable error performance. Furthermore, it was shown employing the configurations of small numbers of subcarriers, large numbers of time slots in GOFDM architecture preserves good spectral efficiency and, besides, yields PAPR even lower than single-carrier FDM scheme. For such configurations, although GFDM transmitter is superior to GOFDM transmitter in terms of PAPR, GFDM MMSE and DSIC/MF receivers suffer from significant cost of numerical complexity and error performance, respectively. In spite of aforementioned advantages, GOFDM has to be precisely evaluated for the case of asynchronous transmitters which is a challenging issue for new 5G applications.

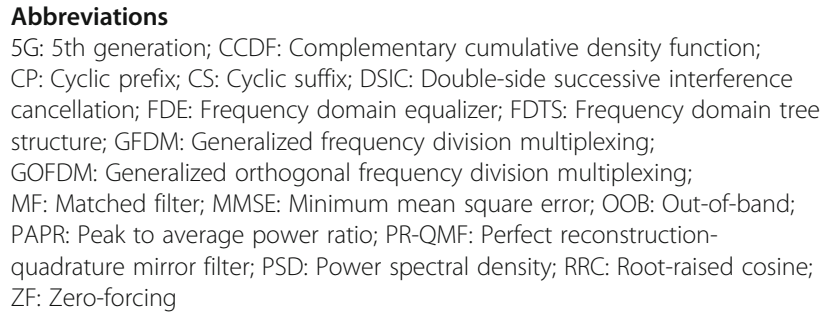

\section{Acknowledgements}

This work was supported in part by a grant from IPM and in part by a grant from the Iran National Science Foundation under grant 95824827.

\section{Funding}

There is no funding for the current research.

Availability of data and materials

The custom codes are available via email to corresponding author.

\section{Authors' contributions}

MHA and BHK have been responsible for providing the idea of the paper. $\mathrm{MHA}$ has also been responsible for implementation of ideas and performing simulations. $\mathrm{MHA}, \mathrm{BHK}$, and $\mathrm{AH}$ have been involved in writing the manuscript and proofreading it.

\section{Authors' information}

Mohammad Hadi Abbaszadeh received his B.Sc. and M.Sc. degrees from Shiraz University and Iran University of Science and Technology, respectively. He is currently pursuing the Ph.D. degree in Tehran Science and Research
Branch, Islamic Azad University, Tehran, Iran. His research interests include MIMO wireless communications and multicarrier modulations.

Babak Hossein Khalaj received his M.Sc. and Ph.D. degrees from Stanford University. At Stanford, he has been among the pioneering team working on adoption of multi-antenna arrays in mobile networks. He has also been a senior member of Advanced Communications Research Institute (ACRI) at Sharif University, Tehran, Iran, and the recipient of Alexander von Humboldt Fellowship in 2007-2008.

Afrooz Haghbin received her M.Sc. and PhD degrees from Tehran University and Tarbiat Modares University, respectively. She is currently with the electrical and computer department of Science and Research Branch in Islamic Azad University, Tehran, Iran, as an assistant professor. Her research interests include MIMO wireless communications, channel coding, precoding, multicarrier modulation, and estimation theory.

\section{Competing interests}

The authors declare that they have no competing interests.

\section{Publisher's Note}

Springer Nature remains neutral with regard to jurisdictional claims in published maps and institutional affiliations.

\section{Author details}

${ }^{1}$ Department of Electrical Engineering, Science and Research Branch, Islamic Azad University, Tehran, Iran. ${ }^{2}$ Department of Electrical Engineering, Sharif University of Technology, Tehran, Iran. ${ }^{3}$ School of Computer Science, Institute for Research in Fundamental Sciences (IPM), Tehran, Iran.

Received: 5 June 2017 Accepted: 21 December 2017

Published online: 12 January 2018

\section{References}

1. SA Fechtel, A Blaickner, Efficient FFT and equalizer implementation for OFDM receivers. IEEE Trans. Consum. Electron. 45(4), 1104-1107 (1999)

2. M Mirahmadi, A Al-Dweik, A Shami, BER reduction of OFDM based broadband communication systems over multipath channels with impulsive noise. IEEE Trans. Commun. 61(11), 4602-4615 (2013)

3. JVD Beek, F Berggren, Out-of-band power suppression in OFDM. IEEE Commun. Lett. 12(9), 609-611 (2008)

4. B Farhang-Boroujeny, OFDM versus filter bank multicarrier. IEEE Signal Process. Mag. 28(3), 92-112 (2011)

5. V Vakilian, T Wild, F Schaich, St Brink, JF Frigon, in 2013 IEEE Globecom Workshops (GC Wkshps). Universal-filtered multi-carrier technique for wireless systems beyond LTE (2013)

6. M Van Eeckhaute, A Bourdoux, P De Doncker, F Horlin, Performance of emerging multi-carrier waveforms for $5 \mathrm{~g}$ asynchronous communications. EURASIP J. Wirel. Commun. Netw. 2017(1), 29 (2017)

7. G Fettweis, M Krondorf, S Bittner, in VTC spring 2009 - IEEE 69th Vehicular Technology Conference. GFDM-generalized frequency division multiplexing (2009)

8. N Michailow, M Matthe, IS Gaspar, AN Caldevilla, LL Mendes, A Festag, G Fettweis, Generalized frequency division multiplexing for 5 th generation cellular networks. IEEE Trans. Commun. 62(9), 3045-3061 (2014)

9. G Wunder, P Jung, M Kasparick, T Wild, F Schaich, Y Chen, ST Brink, I Gaspar, N Michailow, A Festag, L Mendes, N Cassiau, D Ktenas, M Dryjanski, S Pietrzyk, B Eged, P Vago, F Wiedmann, 5gnow: non-orthogonal, asynchronous waveforms for future mobile applications. IEEE Commun. Mag. 52(2), 97-105 (2014)

10. N Michailow, S Krone, M Lentmaier, G Fettweis, in 2012 IEEE Vehicular Technology Conference (VTC Fall). Bit error rate performance of generalized frequency division multiplexing (2012)

11. R Datta, N Michailow, M Lentmaier, G Fettweis, in 2012 IEEE Vehicular Technology Conference (VTC Fall). GFDM interference cancellation for flexible cognitive radio $\mathrm{PHY}$ design (2012)

12. $\mathrm{MH}$ Abbaszadeh, $\mathrm{BH}$ Khalaj, A Haghbin, Error performance analysis for generalized orthogonal frequency division multiplexing. Computers \& Electrical Engineering 61, 139-150 (2017)

13. M Matthe, LL Mendes, G Fettweis, Generalized frequency division multiplexing in a Gabor transform setting. IEEE Commun. Lett. 18(8), 13791382 (2014) 
14. PP Vaidyanathan, Multirate Systems and Filter Banks (Prentice-Hall, Englewood Cliffs, 1993)

15. PP Vaidyanathan, A Kirac, in 1997 IEEE International Conference on Acoustics, Speech, and Signal Process.. Theory of cyclic filter banks (1997)

16. AN Akansu, R.A.H., Multiresolution Signal Decomposition: Transforms, Subbands, and Wavelets (Academic pres, Inc, 1992)

17. AV Oppenheim, RWSchafer, JR Buck, Discrete-Time Signal Processing Upper. Saddle River, N.J., Prentice Hall, (18th ed.). (1999)

18. A Farhang, N Marchetti, LE Doyle, Low-complexity modem design for GFDM. IEEE Trans. Signal Process. 64(6), 1507-1518 (2016)

19. I Gaspar, N Michailow, A Navarro, E Ohlmer, S Krone, G Fettweis, in 2013 IEEE 77th Vehicular Technology Conference (VTC Spring). Low complexity GFDM receiver based on sparse frequency domain processing (2013)

20. H Seung Hee, L Jae Hong, An overview of peak-to-average power ratio reduction techniques for multicarrier transmission. IEEE Wirel. Commun. $12(2), 56-65(2005)$

21. N Michailow, G Fettweis, in 2013 International Symposium on Intelligent Signal Processing and Communication Systems. Low peak-to-average power ratio for next generation cellular systems with generalized frequency division multiplexing (2013)

\section{Submit your manuscript to a SpringerOpen ${ }^{\circ}$ journal and benefit from:}

- Convenient online submission

- Rigorous peer review

- Open access: articles freely available online

- High visibility within the field

- Retaining the copyright to your article

Submit your next manuscript at $\gg$ springeropen.com 